\title{
Shape Modification from Endoscope Images by Regression Analysis
}

\author{
Yuji Iwahori $^{1 *}$, Seiya Tsuda ${ }^{1 *}$, Aili Wang ${ }^{2}$, Robert J. Woodham ${ }^{3}$, M. K. Bhuyan ${ }^{4}$, \\ and Kunio Kasugai ${ }^{5}$ \\ ${ }^{1}$ Dept. of Computer Science, Chubu University, 487-8501 Japan \\ ${ }^{2}$ Dept. of Measurement \& Control Technology and Instrumentations of \\ Heilongjiang, Harbin University of Science and Technology, China \\ ${ }^{3}$ Dept. of Computer Science, University of British Columbia, Canada V6T 1Z4 \\ ${ }^{4}$ Dept. of Electronics and Electrical Engineering, IIT Guwahati, 781039 India \\ ${ }^{5}$ Dept. of Gastroenterology, Aichi Medical University, 480-1195 Japan \\ *Corresponding author: Yuji Iwahori (iwahori@cs.chubu.ac.jp)
}

\begin{abstract}
The VBW (Vogel-Breuß-Weickert) model is proposed as a method to recover 3-D shape under point light source illumination and perspective projection. However, the $V B W$ model recovers relative, not absolute, shape. Here, shape modification is introduced to recover the exact shape. Modification is applied to the output of the VBW model. First, a local brightest point is used to estimate the reflectance parameter from two images obtained with movement of the endoscope camera in depth. A Lambertian sphere image is generated using the estimated reflectance parameter and VBW model is applied for a sphere. Regression analysis is introduced to improve the surface gradients, where linear coefficients can be obtained using true values of gradient parameters with a generated sphere. Depth can then be recovered using the modified gradient parameters. Performance of the proposed approach is confirmed via computer simulation and real experiment.
\end{abstract}

Keywords: Endoscope Image, VBW Model, Regression Analysis, Shape Modification, Reflection Factor

\section{Introduction}

Endoscopy allows medical practitioners to observe the interior of hollow organs and other body cavities in a minimally invasive way. Sometimes, diagnosis requires assessment of the 3-D shape of the observed tissue. For example, the pathological condition of a polyp often is related to its geometrical shape. Medicine is an important area of application of computer vision technology. Many approaches are based on stereo vision [1]. However, the size of the endoscope becomes large and this imposes a burden on the patient. Here, we consider a general purpose endoscope, of the sort still most widely used in medical practice.

Shape recovery from endoscope images is considered. Shape from shading (SFS) [2] and Fast Marching Method (FMM) [3] based SFS approach [4] are proposed. These approaches assume orthographic projection. An extension of FMM to perspective projection is proposed in [5]. Further extension of FMM to both point light source illumination and perspective projection is proposed in [6]. Recent extensions include generating a Lambertian image from the original multiple color images [7-8]. Application of FMM includes solution [9] under oblique illumination using neural network learning [10]. Most of the previous approaches treat the reflectance parameter as a known constant. The problem is that it is impossible to estimate the reflectance parameter from 
only one image. Further, it is also difficult to apply point light source based photometric stereo [11] [12] in the context of endoscopy.

Recently, the Vogel-Breuß-Weickert (VBW) model [13], based on solving the Hamilton-Jacobi equation, has been proposed to recover shape from an image taken under the conditions of point light source illumination and perspective projection. However, the result recovered by the VBW model is relative. VBW gives smaller values for surface gradient and height distribution compared to the true values. That is, it is not possible to apply the VBW model directly to obtain exact shape and size.

This paper proposes a new approach to improve the accuracy of polyp shape determination as absolute size. The proposed approach estimates the reflectance parameter from two images with small camera movement in the depth direction. A Lambertian sphere model is synthesized using the estimated reflectance parameter. The VBW model is applied to the synthesized sphere and shape then is recovered. Surface gradient parameters are obtained by ellipsoid fitting for the height distribution of $\mathrm{Z}$ which is recovered from VBW model. Obtained gradient parameters are used as input for the regression analysis. While the true values of the corresponding gradient parameters are used for the output of regression analysis. The proposed approach is evaluated via computer simulation and real experiments and it is confirmed that the obtained shape is improved.

\section{VBW Model}

The VBW model [13] is proposed as a method to calculate depth (distance from the viewer) under point light source illumination and perspective projection. The method solves the Hamilton-Jacobi equations [14] associated with the models of Faugeras and Prados [15]. Lambertian reflectance is assumed.

The following processing is applied to each point of the image. First, the initial value for the depth $Z_{\text {defaul }}$ is given using Eq.(1) as in [16].

$$
Z_{\text {default }}=-0.5 \log \left(\text { If }^{2}\right)
$$

Where $I$ represents the normalized image intensity and $f$ represents the focal length of the lens.

Next, the combination of gradient parameters which gives the minimum gradient is selected from the difference of depths for neighboring points. The depth, $Z$, is calculated from Eq. (2) and the process is repeated until the $Z$ values converge. Here, $(x, y)$ are the image coordinates, $\Delta t$ is the change in time, $(m, n)$ is the minimum gradient for $(x, y)$ directions, and $Q=f / \sqrt{x^{2}+y^{2}+f^{2}}$ is the coefficient of the perspective projection.

$$
\begin{aligned}
& Z(x, y)=Z(x, y)+\Delta t \exp (-2 Z(x, y)) \\
& -\Delta t\left(\frac{I f^{2}}{Q} \sqrt{f^{2}\left(m(x)^{2}+n(y)^{2}\right)+(x m(x)+y n(y))^{2}+Q^{2}}\right)
\end{aligned}
$$

Here, it is noted that the shape obtained with the VBW model is given in a relative scale, not an absolute one. The obtained result gives smaller values for surface gradients than the actual gradient values.

\section{Proposed Approach}

\subsection{Estimating Reflectance Parameter}

When uniform Lambertian reflectance and point light source are assumed, image intensity depends on the dot product of surface normal vector and the light source direction vector subject to the inverse square law for illuminance. 
Measured intensity at each surface point is determined by Eq. (3).

$$
E=C \frac{(\mathbf{s} \cdot \mathbf{n})}{r^{2}}
$$

Where $E$ is image intensity, $\mathbf{s}$ is a unit vector towards the point light source, $\mathbf{n}$ is a unit surface normal vector, and $r$ is the distance between the light source and surface point.

The proposed approach estimates the value of the reflectance parameter, $C$, using two images acquired with a small camera movement in the depth direction. It is assumed that $C$ is constant for all points on the Lambertian surface. Regarding geometry, it is assumed that both the point light source and the optical center of lens are co-located at the origin of the $(X, Y, Z)$ world coordinate system. Perspective projection is assumed.

The actual endoscope image has the color textures and specular reflectance. Using the approach proposed by [17] the original input endoscope image is converted into one that satisfies the assumptions of a uniform Lambertian gray scale image.

The procedure to estimate $C$ is as follows.

Step 1 . If the value of $C$ is given, depth $Z$ is uniquely calculated and determined at the point with the local maximum intensity [18]. At this point, the surface normal vector and the light source direction vector are aligned and produce the local maximum intensity for that value of $C$.

Step 2. For camera movement, $\Delta Z$, in the $Z$ direction, two images are used and the difference in $Z, Z_{d i f f}$, at the local maximum intensity points in each image is calculated. Here the camera movement, $\Delta Z$, is assumed to be known.

Step 3. Let $f(C)$ be the error between $\Delta Z$ and $Z_{\text {diff }} . f(C)$ represents an objective function to be minimized to estimate the correct value of $C$. That is, the value of $C$ is the one that minimizes $f(C)$ given in Eq. (4).

$$
f(C)=\left(\Delta Z-Z_{\text {diff }}(x, y)\right)^{2}
$$

\subsection{Regression Analysis for Shape Modification}

The size and shape recovered by the VBW model are relative. VBW gives smaller values for surface gradient and depth compared to the true values. Here, modification of surface gradient and improvement of the recovered shape are considered. First, the surface gradient at each point is modified by a regression analysis. Then the depth is modified using the estimated reflectance parameter $C$ and modified surface gradient parameters $(p, q)=\left(\frac{\partial Z}{\partial X}, \frac{\partial Z}{\partial Y}\right)$ by regression analysis. Here, the regression analysis estimates the linear coefficients of regression model and modifies each of the surface gradient parameters for the recovered result by VBW model. Using the estimated $C$, a sphere image is synthesized with uniform Lambertian reflectance. The VBW model is applied to this synthesized sphere. Ellipsoid is assumed under the consideration to treat a sphere. Equation is written as Eq.(5) where $x$ and $y$ represent the image coordinates corresponding to the world coordinates $(X, Y), z$ represents the depth (height) information) obtained from VBW. Coefficients $S, T$, $U$ can be obtained using $n$ sampled points of ellipsoid using linear least squares as shown in Eq.(6) to Eq.(8). Transforming Eq.(5) gives Eq.(9) and gradient parameters $(\mathrm{p}, \mathrm{q})$ are given by Eq.(10).

$$
S x^{2}+T y^{2}+U z^{2}=1
$$




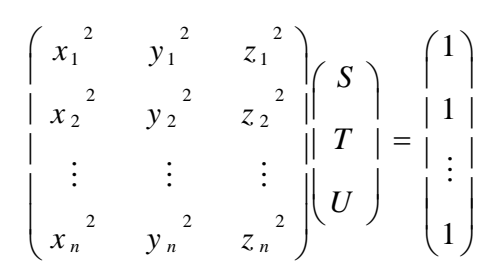

$$
\begin{aligned}
& L=\left(\begin{array}{ccc}
x_{1}{ }^{2} & y_{1}{ }^{2} & z_{1}{ }^{2} \\
x_{2}{ }^{2} & y_{2}{ }^{2} & z_{2}{ }^{2} \\
\vdots & \vdots & \vdots \\
x_{n}{ }^{2} & y_{n}{ }^{2} & z_{n}{ }^{2}
\end{array}\right), m=\left(\begin{array}{c}
S \\
T
\end{array} \mid, n=\left(\begin{array}{l}
1 \\
1
\end{array} \mid\right.\right. \\
& m=\left(L^{T} \cdot L\right)^{-1} \cdot L^{T} \cdot n \\
& z=\sqrt{\frac{1-S x^{2}-T y^{2}}{U}} \\
& p=\frac{\partial z}{\partial x}=\frac{1}{2}\left(\frac{1-S x^{2}-T y^{2}}{U}\right)^{-\frac{1}{2}} \cdot\left(-\frac{2 S x}{U}\right)=-\frac{S x}{U}\left(\frac{1-S x^{2}-T y^{2}}{U}\right)^{-\frac{1}{2}}, \\
& q=\frac{\partial z}{\partial y}=\frac{1}{2}\left(\frac{1-S x^{2}-T y^{2}}{U}\right)^{-\frac{1}{2}} \cdot\left(-\frac{2 T y}{U}\right)=-\frac{T y}{U}\left(\frac{1-S x^{2}-T y^{2}}{U}\right)^{-\frac{1}{2}}
\end{aligned}
$$

The estimated gradients, $(p, q)$, and the corresponding true gradients for the synthesized sphere, $(p, q)$, are given respectively as input vectors and output vectors to the regression analysis. Suppose when we assume the $3^{\text {rd }}$ order polynomial for the relation between the original gradient parameter and the corresponding true gradient parameter, the regression model becomes Eq.(11) and four coefficients $a$ to $d$ are obtained using linear least squares of Eq.(12) to Eq.(14) with $n$ samples points on the Lambertian sphere.

$$
\begin{gathered}
y=a x^{3}+b x^{2}+c x+d \\
A x=y
\end{gathered}
$$

Where

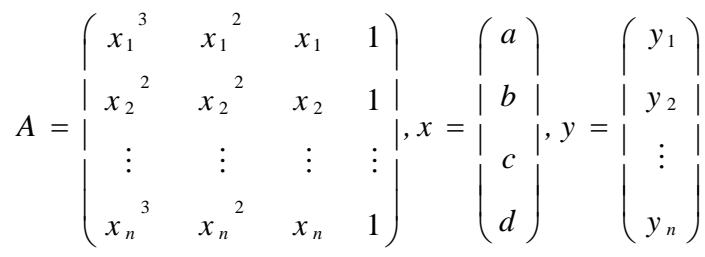

$$
\begin{aligned}
& x=\left(A^{T} A\right)^{-1} A^{T} y
\end{aligned}
$$

Here $x$ is assumed to be gradient parameter $p$ or $q$, and $\mathrm{y}$ is assumed to be modified gradient parameter $p$ or $q$. Substituting coefficients $a$ to $d$ into Eq.(11) can obtain the modified gradient parameters $p$ or $q$. Gradient parameter $p$ and $q$ are obtained with numerical difference taken for the recovered result $z$ of VBW. For the 
real endoscope image, these parameters are used to this regression model for the modification.

In the case of endoscope images, preprocessing is used to remove specular points and to generate a uniform Lambertian image based on [17].

Next, the VBW model is applied to the this Lambertian image and the gradients, $(p, q)$, are estimated from the obtained $Z$ distribution.

The estimated gradients, $(p, q)$, are input to the regression analysis and modified estimates of $(p, q)$ are obtained as output from the regression analysis.

Recall that the reflectance parameter, $C$, is estimated from $f(C)$, based on two images obtained by small movement of endoscope in the $Z$ direction.

The depth, $Z$, is calculated and updated by Eq.(15) using the modified gradients, $(p, q)$, and the estimated $C$, where Eq.(15) also is the original equation developed in [6].

$$
Z=\sqrt{\frac{C V(-p x-q y+f)}{E\left(p^{2}+q^{2}+1\right)^{\frac{1}{2}}}}
$$

Again, $(p, q)=(\partial Z / \partial X, \partial Z / \partial Y), E$ represents image intensity, $f$ represents the focal length of the lens and $V=\frac{f^{2}}{\left(x^{2}+y^{2}+f^{2}\right)^{\frac{3}{2}}}$ (we call the coefficient of perspective projection).

\section{Experimental Results}

\subsection{Regression Analysis}

Sphere image is synthesized with the center coordinate $(0,0,15)$ and radius $\mathrm{r}=5 \mathrm{~mm}$, focal length $\mathrm{f}=10 \mathrm{~mm}$ and image size $=9 \mathrm{~mm}$ for each. Gradient parameters $(\mathrm{p}, \mathrm{q})$ are obtained with ellipsoid fitting for the obtained height distribution of $\mathrm{z}$. This ellipsoid fitting is important to estimate reliable coefficients of $n$-th order polynomial. Ellipsoid fitting can also correspond to the difference of aspect ratio of imaging device.

VBW model was applied to a sphere image and ellipsoid fitting was applied to the result by VBW. Obtained gradient parameters were given as input data while the corresponding true gradient parameters were given as output data of regression analysis. $3^{\text {rd }}$ order regression analysis was applied and the coefficients $a$ to $h$ were obtained as $a=$ $39.7316, b=-3.8192 \mathrm{e}-14, \quad c=6.9932, d=1.6263 \mathrm{e}-18, \quad e=-39.7316, f=9.0206 \mathrm{e}-13$, $g=6.9932, h=-2.6102 \mathrm{e}-17$, respectively. These coefficients were used in Eq.(11) and gradient parameters were modified. Cross section of true parameter $p$ is shown in Figure1 (a), parameter $\mathrm{p}$ before modification is shown in Figure 1 (b), while parameter $\mathrm{p}$ after modification using regression analysis is shown in Figure 1 (c).

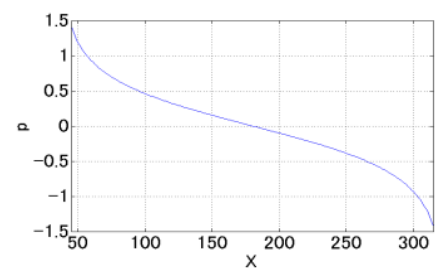

(a) True $p$

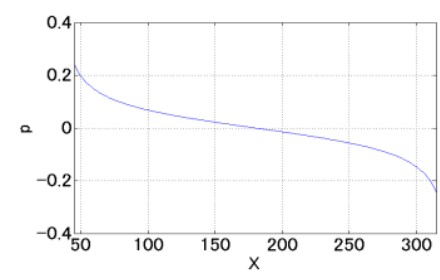

(b) $p$ Obtained by VBW

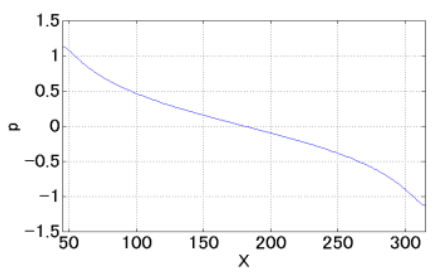

(c) Modified $p$

Figure 1. Cross Section of Surface Gradient Parameter 
It is shown that regression analysis performed good modification of gradient parameters and it is confirmed that the regression analysis using a Lambertian sphere works well.

Other order number of $n$ in polynomial used in regression analysis is also investigated via simulation. Mean error was evaluated for the height $z$ in each case with substituting the modified $(p, q)$ into Eq.(15). The result by VBW and that after each case of $1^{\text {st }}$ to $5^{\text {th }}$ regression analysis are shown in Table 1, where the number of percentage is the proportion to the radius $\mathrm{r}=5 \mathrm{~mm}$ of a sphere.

\section{Table 1. Mean Error of $Z$ [mm]}

\begin{tabular}{|c|c|c|c|c|c|}
\hline $\begin{array}{c}\text { VBW } \\
\text { Model }\end{array}$ & $\begin{array}{c}\text { 1st-order } \\
\text { Regression }\end{array}$ & $\begin{array}{c}\text { 2nd-order } \\
\text { Regression }\end{array}$ & $\begin{array}{c}\text { 3rd-order } \\
\text { Regression }\end{array}$ & $\begin{array}{c}\text { 4th-order } \\
\text { Regression }\end{array}$ & $\begin{array}{c}\text { 5th-order } \\
\text { Regression }\end{array}$ \\
\hline $3.6343(72.5 \%)$ & $0.3635(7.3 \%)$ & $0.3635(7.3 \%)$ & $0.3221(6.4 \%)$ & $0.3203(6.4 \%)$ & $0.3203(6.4 \%)$ \\
\hline
\end{tabular}

Table 1 shows every case of regression analysis with different order polynomials gives improvement than the result of VBW. $1^{\text {st }}$ and $2^{\text {nd }}$ order regression gave some difference but $3^{\text {rd, }} 4^{\text {th }}$ and $5^{\text {th }}$ order regressions did not give much difference. From these observations, $3^{\text {rd }}$ order regression analysis is useful and recommended to the modification.

\subsection{Computer Simulation}

Computer simulation was performed for two synthesized images with a small difference $z$ to confirm the performance of the regression analysis.

Synthesized cosine curved surfaces were used, one with center located at coordinates $(0,0,12)$ and the other with center at $(0,0,15)$. Common to both, the reflectance parameter, $C$, is 120 , the focal length $f$ is $10 \mathrm{~mm}$ and the waveform cycle is $4 \mathrm{~mm}$ and the \pm amplitude is $1 \mathrm{~mm}$. Image size is $5 \mathrm{~mm} \times 5 \mathrm{~mm}$ and pixel size is $256 \times 256$ pixels. The synthesized image whose center is located at $(0,0,12)$ is shown in Figure 2 (a) and the one with center located at $(0,0,15)$ is shown in Figure $2(b)$.

The reflectance parameter $C$ was estimated according to the proposed method and gradient was modified using $3^{\text {rd }}$ order regression analysis in section 4.1. After gradient parameters, $(p, q)$ were modified, depth $Z$ were updated using Eq.(15). The graph of the objective function, $f(C)$, is shown in Figure 3 and the true depth is shown in Figure4 (a). The estimated $C$ was 119 (compared to the true value of 120). The estimated $Z_{\text {diff }}$ was 2.9953 (compared to the true value of 3 ).

The result recovered by VBW for Figure2(a) is shown in Figure4(b). The modified values of depth, using the regression analysis and Eq. (15), are shown in Figure4(c). Table. 2 gives the mean errors in surface gradient and depth estimation. The percentages given in the $Z$ column represent the error relative to the amplitude of maximum minimum depth $(=2 \mathrm{~mm})$ of the cosine synthesized function.

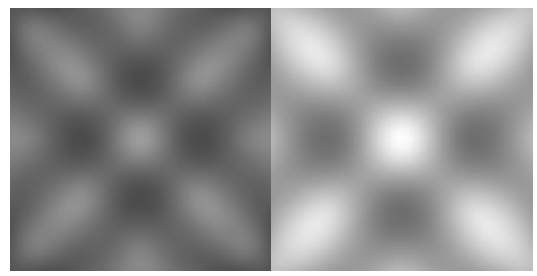

(a) Center: $(0,0,12) \quad$ (b) Center : $(0,0,15)$

Figure 2. Cosine Model 


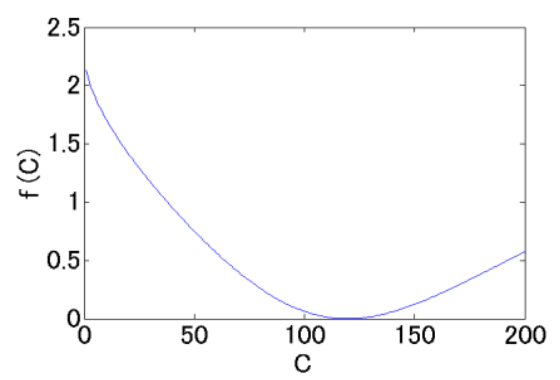

Figure 3. Objective Function $f(C)$

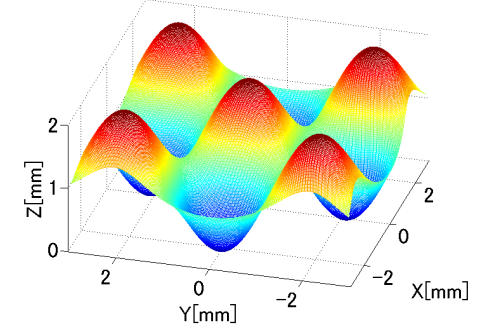

(a) True $Z$

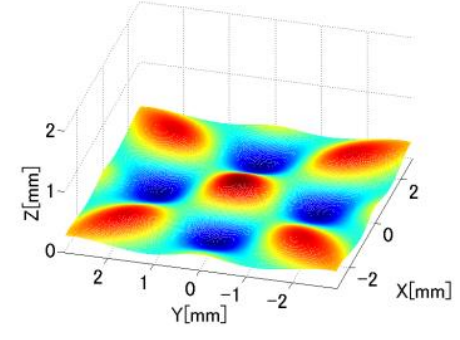

(b) $Z$ by VBW

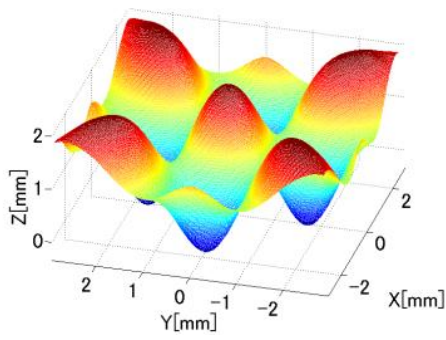

(c) Modified $Z$

Figure 4. Results

Table 2. Mean Error

\begin{tabular}{|c|c|c|c|}
\hline & $p$ & $q$ & $Z[\mathrm{~mm}]$ \\
\hline VBW & 23.0406 & 23.0406 & $0.8611(43.1 \%)$ \\
\hline Proposed & 0.3259 & 0.3259 & $0.1823(9.1 \%)$ \\
\hline
\end{tabular}

It is confirmed that the shape is improved with modification in the proposed approach from Figure4. This means that regression analysis modified $(p, q)$ for each point and $\mathrm{Z}$ are modified correctly. In Table 2, Depth estimation is improved to a mean error of $9.1 \%$ from $43.1 \%$. Regression analysis improved shape for an object with absolute size and shape. Total processing time was 7 seconds.

Another experiment was performed under the following assumptions. The reflectance factor, $C$, is 590 , the focal length, $f$, is $10 \mathrm{~mm}$ and the object is a sphere with radius $5 \mathrm{~mm}$. The centers for two positions of the sphere were set at $(0,0,15)$ and $(0,0,17)$ respectively. The image size was $9 \mathrm{~mm} \times 9 \mathrm{~mm}$ with pixel size $360 \times 360$ pixels. Here, $4 \%$ Gaussian noise (mean 0, variance 0.02, standard deviation 0.14142) is added to each of the two input images. Evaluations for 6\% (mean 0, variance 0.03, standard deviation 0.17320 ) and $10 \%$ (mean 0, variance 0.05 , standard deviation 0.22360) Gaussian noise are shown in Table 3 , as well.

Table 3. Mean Error of $Z$ for Different Gaussian Noise

\begin{tabular}{|c|c|c|c|}
\hline & $4 \%$ & $6 \%$ & $10 \%$ \\
\hline VBW & $3.2007(64.0 \%)$ & $3.2917(65.8 \%)$ & $3.3607(67.2 \%)$ \\
\hline Proposed & $0.4380(8.8 \%)$ & $0.4817(9.6 \%)$ & $0.5630(11.3 \%)$ \\
\hline
\end{tabular}

In all three cases, Gaussian noise of $4 \%, 6 \%$ and 10\%, the proposed approach reduced the mean error in $Z$ significantly compared to the original VBW model. This suggests modification using the regression analysis is robust to noise and is applicable to real imaging situations, including endoscopy. 


\subsection{Real Image Experiments}

Two endoscope images obtained with camera movement in the $Z$ direction are used in the experiments. VBW was applied to one of the images which was first converted to a uniform Lambertian image. After estimating the reflectance parameter $C$, coefficients of regression model were obtained using a sphere model with the estimated value of $C$.

Surface gradients, $(p, q)$, were modified with the $3^{\text {rd }}$ order regression model then depth, $Z$, was updated at each image point. The focal length, $f=10 \mathrm{~mm}$, the image size $5 \mathrm{~mm} \times$ $5 \mathrm{~mm}$, and camera movement, $\Delta Z=3 \mathrm{~mm}$, were assigned to the same known values as those in the computer simulation.

The two endoscope images are shown in Figure5(a) and (b). The generated Lambertian images are shown in Figure5(c) and (d), respectively. The objective function, $f(C)$, is shown in Figure6. The result from the VBW model is shown in Figure7(a) and the modified result is shown in Figure7(b).

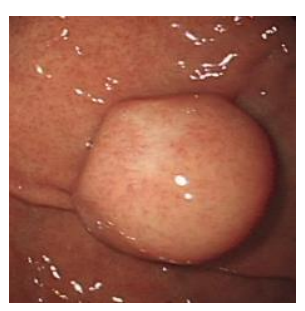

(a) Endoscope 1

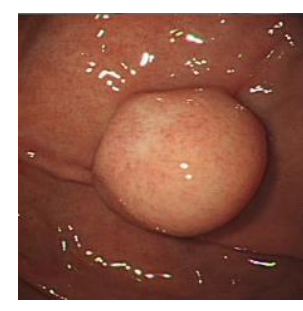

(b) Endoscope 2

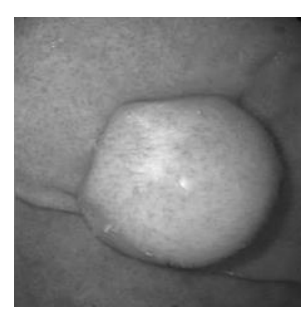

(c) Lambert 1

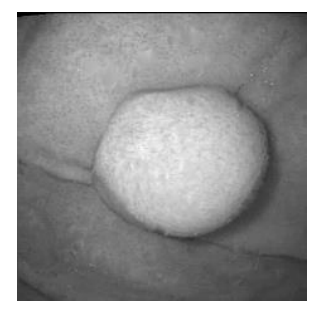

(d) Lambert 2

Figure 5. Endoscope Image and Generating Lambert Image

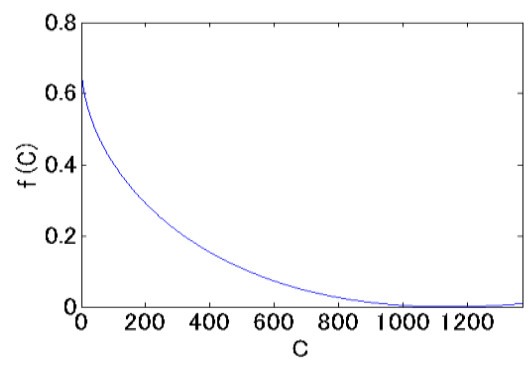

Figure 6. Objective Function $f(C)$

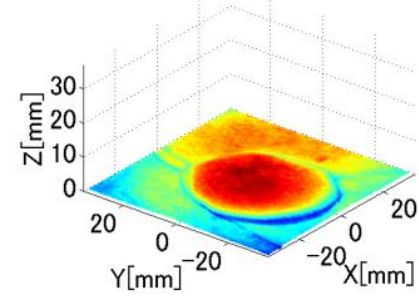

(a) $Z$ by VBW

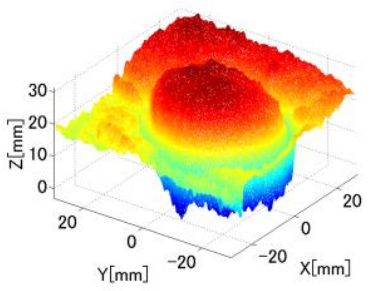

(b) Modified $Z$

Figure 7. Result for Endoscope Images

The estimated value of the reflectance parameter, $C$, was 1141 from Figure6. The difference in depth, $Z$, at the local maximum point was $1[\mathrm{~mm}]$ for the camera movement $Z_{\text {diff }}$ between two images. In Figure5(c)(d), specularities were removed compared to Figure5(a)(b). The converted images are gray scale with the appearance of uniform 
reflectance. Figure7(b) gives a larger depth range than Figure7(a). This suggests depth estimation is improved. The size of the polyp was $1 \mathrm{~cm}$ and the processing time for shape modification was 12 seconds.

Although quantitative evaluation is difficult, medical doctors with experience in endoscopy qualitatively evaluated the result to confirm its correctness. Different values of the reflectance parameter, $C$, were estimated in different experimental environments. The absolute size of a polyp is estimated based on the estimated value of $C$. Accurate values of $C$ lead to accurate estimation of the size of the polyp. The estimated polyp sizes were seen as reasonable by the medical doctor. This qualitatively confirms that the proposed approach is effective in real endoscopy.

Another experiment was done for the endoscope images shown in Figure8(a)(b). The generated gray scale Lambertian images are shown in Figure8(c)(d), respectively. Here the focal length is $10 \mathrm{~mm}$, image size is $5 \mathrm{~mm} \times 5 \mathrm{~mm}$, pixel size is $256 \times 256$ and $\Delta Z$ was set to be $10 \mathrm{~mm}$. The graph of $f(C)$ is shown in Figure9. The VBW result for Figure8(d) is shown in Figure10(a), while that for the proposed approach is shown in Figure10(b).
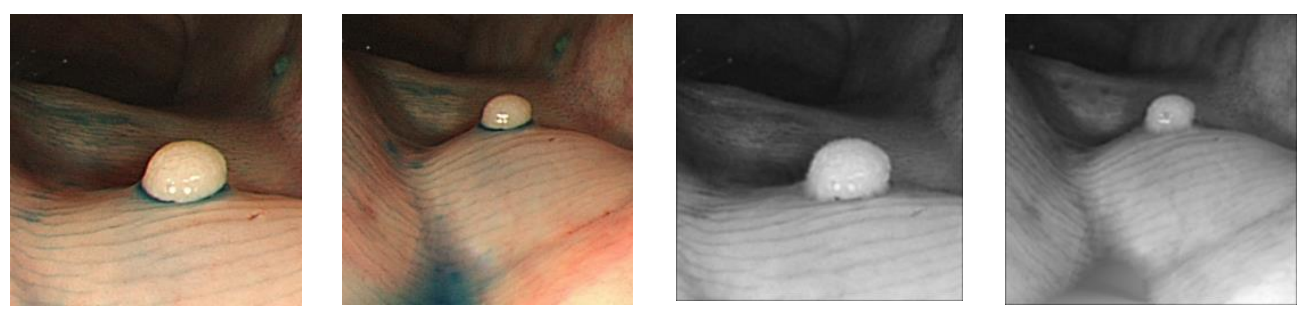

(a) Endoscope 1 (c) Lambert 1

(d) Lambert 2

\section{Figure 8. Endoscope Image and Generating Lambert Image}

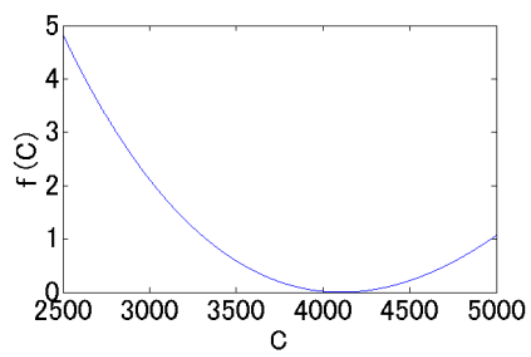

Figure 9. Objective Function $f(C)$

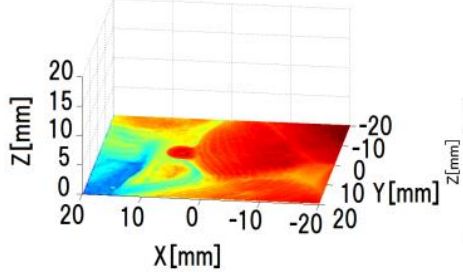

(a) $Z$ by VBW

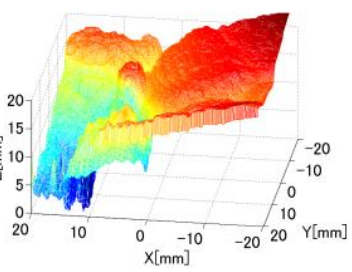

(b) Modified Z

Figure 10. Result for Endoscope Images

$C$ was estimated as 4108 from Figure9. Figure10(b) shows greater depth amplitude compared to Figure10(a). The estimated size of the polyp was about 5mm. This corresponds to the convex and concave shape estimation based on a stain solution. Total processing time was 12 seconds. 


\section{Conclusion}

This paper proposed a new approach to improve the accuracy of absolute size and shape determination of polyps observed in endoscope images.

Coefficients of regression model are obtained for a Lambertian sphere and surface gradient parameters were modified for the result where VBW model is applied for endoscope images. Thus VBW model was used to estimate a baseline shape. Modification of gradients with the regression analysis improved the accuracy of shape and absolute size was obtained via modification. Estimation of the reflectance parameter $C$ was achieved under the assumption that two images are acquired via small camera movement in the depth, $Z$, direction. The approach was evaluated both in computer simulation and with real endoscope images. Results confirm that the approach improves the accuracy of recovered shape to within error ranges that are practical for polyp analysis in endoscopy. Further research includes an extension to endoscope video with scene understanding and realizing faster processing time.

\section{Acknowledgment}

Iwahori's research is supported by Japan Society for the Promotion of Science (JSPS) Grant-in-Aid for Scientific Research (C) (26330210) and Chubu University Grant. Woodham's research is supported by the Natural Sciences and Engineering Research Council (NSERC). The authors would like to thank Yuki Hanai for his experimental help and the related member for useful discussions in this paper.

\section{References}

[1] T. Thormaehlen, H. Broszio and P. N. Meier, "Three-Dimensional Endoscopy", Falk Symposium, (2001), pp. 199-212.

[2] B. K. P. Horn, "Obtaining Shape from Shading Information", The Psychology of Computer Vision, Winston, P. H. (Ed.), Mc Graw-Hill, (1975), pp. 115-155.

[3] J. A. Sethian, "A Fast Marching Level Set Method for Monotonically Advancing Fronts", Proceedings of the National Academy of Sciences of the United States of America (PNAS U.S.), vol. 93, no. 4, (1996), pp. 1591- 1593.

[4] R. Kimmel and J. A. Sethian, "Optimal Algorithm for Shape from Shading and Path Planning", Journal of Mathematical Imaging and Vision (JMIV), vol. 14, no. 3, (2001), pp. 237-244.

[5] S. Y. Yuen, Y. Y. Tsui and C. K. Chow, "A fast marching formulation of perspective shape from shading under frontal illumination," Pattern Recognition Letters, vol. 28, no. 7, (2007), pp. 806-824.

[6] Y. Iwahori, K. Iwai, R. J. Woodham, H. Kawanaka, S. Fukui and K. Kasugai, “ Extending Fast Marching Method under Point Light Source Illumination and Perspective Projection”, ICPR, (2010), pp. 1650-1653.

[7] Y. Ding, Y. Iwahori, T. Nakamura, L. He, R. J. Woodham and H. Itoh, "Shape Recovery of Color Textured Object Using Fast Marching Method via Self-Calibration”, EUVIP, (2010), pp. 92-96.

[8] D. R. Neog, Y. Iwahori, M. K. Bhuyan, R. J. Woodham and K. Kasugai, "Shape from an Endoscope Image Using Extended Fast Marching Method", Proc. of IICAI-11, (2011), pp. 1006-1015.

[9] Y. Iwahori, K. Shibata, H. Kawanaka, K. Funahashi, R. J. Woodham and Y. Adachi, "Shape from SEM Image Using Fast Marching Method and Intensity Modification by Neural Network", Recent Advances in Knowledge-based Paradigms and Applications, Advances in Intelligent Systems and Computing 234, Springer, no. 5, (2014), pp. 73-86.

[10] Y. Ding, Y. Iwahori, T. Nakamura, R. J. Woodham, L. He and H. Itoh, "Self-calibration and Image Rendering Using RBF Neural Network", KES, vol. 5712, (2009), pp. 705-712.

[11] Y. Iwahori, H. Sugie and N. Ishii, "Reconstructing Shape from Shading Images under Point Light Source Illumination”, ICPR, v.1, (1990), pp. 83-87.

[12] Y. Iwahori and R. Woodham, "Neural Network based Photometric Stereo with a Nearby Rotational Moving Light Source”, IEICE Trans. Info. and Syst., vol. E80-D, no. 9, (1977), pp. 948-957.

[13] O. Vogel, M. Breuß and J. Weickert, "A Direct Numerical Approach to Perspective Shape-fromShading”, Vision Modeling and Visualization (VMV), (2007), pp. 91-100.

[14] S. H. Benton, "The Hamilton-Jacobi Equation: A Global Approach", Academic Press, vol. 131, (1977).

[15] E. Prados and O. D. Faugeras, "Unifying Approaches and Removing Unrealistic Assumptions in Shape from Shading: Mathematics Can Help”, ECCV, (2004). 
[16] E. Prados and O. Faugeras, "Shape From Shading: a well-posed problem?” CVPR, (2005), pp. 870-877.

[17] Y. Shimasaki, Y. Iwahori, D. R. Neog, R. J. Woodham and M. K. Bhuyan, "Generating Lambertian Image with Uniform Reflectance for Endoscope Image”, IWAIT, 1C-2 (Computer Vision 1), (2013), pp. 60-65.

[18] K. Tatematsu, Y. Iwahori, T. Nakamura, S. Fukui, R. J. Woodham and K. Kasugai, "Shape from Endoscope Image based on Photometric and Geometric Constraints", KES, Procedia Computer Science, Elsevier, vol. 22, (2013), pp. 1285-1293.

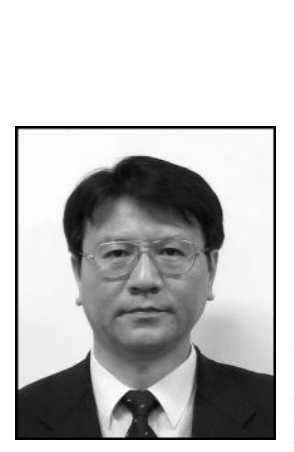

\section{Authors}

Yuji Iwahori, received B.S. degree from Dept. of Computer Science, Nagoya Institute of Technology, M.S. degree and Ph.D. degree from the Dept. of Electrical and Electronics, Tokyo Institute of Technology in 1985 and 1988, respectively. He joined Nagoya Institute of Technology as a research associate in 1988 and he became a professor in 2002. He has joined Chubu University as a professor since 2004. In the meanwhile, he has been a visiting researcher of UBC Computer Science, Canada since 1991. He has been a research collaborator with IIT Guwahati. His research interests include Computer Vision, Image Recognition, Neural Network and Application of Artificial Intelligence. He got KES 2008 Best Paper Award and KES 2013 Best Paper Award from KES International.

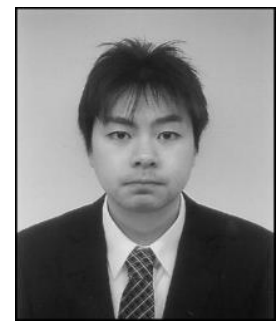

Seiya Tsuda, graduated from Dept. of Computer Science, Chubu University, Japan in 2013. He is now a graduate student of Chubu University. He is a member of IEICE. His research interest includes Computer Vision, Medical Image Processing, Virtual Reality and Augmented Reality.

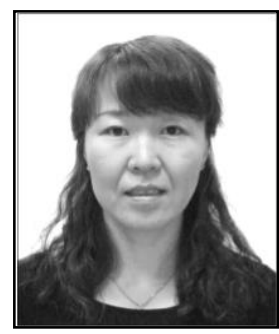

Wang Aili, received B.S. degree from Dept. of Electronic and Communication Engineering, M.S. and Ph.D. degree from Dept. Information and Communication Engineering, Harbin Institute of Technology in 2002, 2004 and 2008, respectively. She joined Harbin University of Science and Technology as a assistant in 2004 and she became a associate professor in 2010. She has been a visiting professor to Chubu University, Japan since 2014. Her research interests include image super-resolution, image fusion and object tracking.

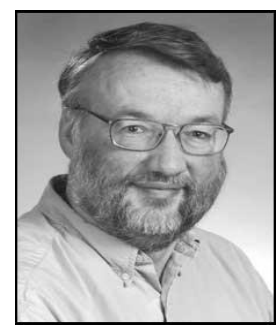

Robert J. Woodham, received B.A. degree in Mathematics from the University of Western Ontario in 1971. Then he received S.M. and E.E. degrees in Electrical Engineering from MIT in 1974. He received Ph.D. degree in Electrical Engineering and Computer Science from MIT in 1977. He was a post-doctoral research scientist at MIT until July 1978. In August 1978, he became a faculty member at UBC. He is currently a professor of Computer Science at UBC. His principal research interest is computational vison, including shape from shading, photometric stereo, optical flow and orientationbased representations of shape. 


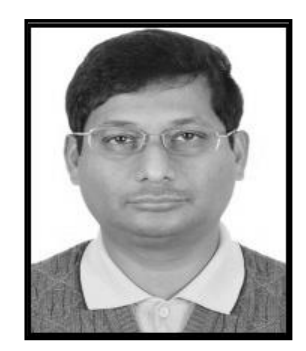

M. K. Bhuyan, M.K. Bhuyan is an Associate Professor in the Department of Electronics and Electrical Engineering, Indian Institute of Technology (IIT) Guwahati, India. He has a Ph.D. degree in Electronics and Communication Engineering from Indian Institute of Technology Guwahati, India. He did his postdoctoral research in the school of Information Technology and Electrical Engineering, University of Queensland, Australia, and then in National ICT, Brisbane, Australia. Earlier, he worked as an Assistant Professor in the department of Electrical Engineering, IIT Roorkee, India. He also worked as a visiting Associate Professor in Indiana University-Purdue University, USA. His research interests include Image/Video Processing, Computer Vision and Human Computer Interactions. He got the National Award for "Best Applied Research/Technological Innovation Aimed at Improving the Life of Persons with Disabilities" by Government of India in 2013.

Kunio Kasugai, received B.M. degree from Faculty of Medicine, Nagoya City University in 1985 and Ph.D. in Medicine from Nagoya City University in 1996. He joined Aichi Medical University as a lecturer in 1997. He became an associate professor of Dept. of Gastroenterology, Aichi Medical University in 2004. In the meanwhile, he became a visiting researcher of Michigan University in 1999. He has been a professor of Dept. of Gastroenterology, Aichi Medical University since 2007. He is now a vice director of hospital of Aichi Medical University. 